Seit Oktober des letzten Jahres kann der neue Wirkstoff Aclidiuniumbromid Patienten mit COPD verordnet werden. Der langwirksame Muskarin-Rezeptor-Antagonist (LAMA) wird mit einem neuen, technisch hoch entwickelten Multidosis-Pulverinhalator (Abb. 1) appliziert.

Aclidiniumbromid wird $2 \times$ täglich, morgens und abends in der Dosierung von je $400 \mu \mathrm{g}$ inhaliert. Die Wirkung tritt meist innerhalb von 30 Minuten nach der ersten Inhalation ein und hält aufgrund der Zweimalgabe häufig bis in den Morgen an [1,2].

Aclidiniumbromid verbessert die Lungenfunktion im Vergleich zu Placebo signifikant. So verbesserte sich das FEV1 vor der morgendlichen Inhalation (Trough-FEV1) innerhalb von 24 Wochen um $128 \mathrm{ml}(+/-22$ $\mathrm{ml}$ SD) im Vergleich zu Placebo ( $\mathrm{p}<$ 0,0001) [2]. Das maximale FEV1 (Peak-
FEV1) nach der Inhalation betrug $209 \mathrm{ml}$ (+/- $24 \mathrm{ml} \mathrm{SD})$ [2].

Mit Aclidiniumbromid behandelte Patienten benötigten weniger Notfallmedikamente als die in der Placebogruppe. Auch COPD-Symptome wie Atemnot besserten sich signifikant $[1,2]$.

Die Therapie mit Aclidiniumbromid wird gut vertragen. Die in den Studien dokumentierten unerwünschten Ereignisse waren meist leicht bis moderat $[1,2]$. Am häufigsten traten Kopfschmerzen $(6,6 \%)$ und Entzündungen des Nasenrachenraums (5,5\%) auf [3]. Die Inzidenz von anticholinergen Nebenwirkungen war mit der in der Placebogruppe vergleichbar $[1,2]$.

Der Wirkstoff wird mit Hilfe des Trockenpulverinhalators Genuair inhaliert, einer Weiterentwicklung des Novolizers. Es handelt sich um einen atemzuggesteuerten Multidosis-Inhalator, der in Deutschland entwickelt und patentiert wurde. Der Inhala-

Abb. 1. MultidosisPulverinhalator zur Applikation von Aclidiniumbromid. tor wurde so konzipiert, dass bei korrekter Inhalation Rückmeldungen erfolgen und schwierige und fehleranfällige Prozesse bei der Bedienung entfallen. Außerdem ist es nicht möglich aus einem leeren Inhalator zu inhalieren, denn nach Inhalation der letzten Dosis arretiert die Taste, mit der man den Wirkstoff zur Inhalation freisetzt.

Aclidiniumbromid $\left(\right.$ Bretaris $^{\circledR}$ Genuair $^{\circledR}$ ) erwies sich aufgrund der schnell einsetzenden und bei Zweimalgabe andauernden Wirksamkeit und der geringen Rate anticholinergischer sowie anderer unerwünschter Wirkungen als effektive und gut verträgliche Therapiestrategie für Patienten mit COPD.

\section{Literatur}

1 Kerwin EM, D'Urzo AD, Gelb AF, Lakkis H, Gil GE, Caracta CF, ACCORD I study investigators: Efficacy and safety of a 12 -week treatment with twice-daily aclidinium bromide in COPD patients (ACCORD COPD I) COPD 2012;9(2):90-101.

2 Jones PW, Singh D, Bateman ED, Agusti A, Lamarca R, de Miquel G, Segarra R, Caracta C, Garcia Gil E: Efficacy and safety of twice-daily aclidinium bromide in COPD patients: The ATTAIN study. Eur Respir J 2012;40(4):830-6.

3 Fachinformation Bretaris ${ }^{\circledR}$ Genuair $^{\circledR}$; Stand Juli 2012 www.ema.europa.eu/docs/de_DE/document_library/ EPAR_-_Product_Information/human/002211/ WC500132661.pdf

Weitere Informationen bei

Waggener Edstrom Worldwide

Dr. Maren Mundt

Sandstraße 33, 80335 München

Tel. +4989 628175-32

Mmundt@waggeneredstrom.com

\title{
PharmaTicker+++ PharmaTicker+++ PharmaTicker+++ PharmaTicker+++
}

Pfizer. Die Europäische Kommission hat Xalkori ${ }^{\circledR}$ (Wirkstoff: Crizotinib) eine bedingte Zulassung («Conditional Marketing Authorization») zur Behandlung von erwachsenen Patienten mit vorbehandeltem ALK-positivem fortgeschrittenem nicht-kleinzelligem Lungenkarzinom (NSCLC) erteilt. Crizotinib ist ein oraler Inhibitor der Anaplastischen-Lymphom-Kinase (ALK) und die erste Therapieoption, die spezifisch auf die Konsequenzen von ALK-Genrearrangements ausgerichtet ist.

Pfizer Deutschland GmbH

Dr. Achim Janik

presse@pfizer.com
Chiesi. Mit FOSTER ${ }^{\circledR}$ NEXThaler ${ }^{\circledR}$, dem ersten und einzigen Trockenpulver-Inhalator mit extrafeinen Teilchen, erweitern sich ab März 2013 die Therapiemöglichkeiten beim Asthma bronchiale. Das neue Device überzeugt durch seine einfache Handhabung. Neben dem bewährten FOSTER ${ }^{\circledR}$ Dosieraerosol steht somit eine weitere extrafeine Fix-Kombination aus Beclometason und Formoterol zur Verfügung, die es ermöglicht das Device entsprechend den Vorlieben der Patienten zu wählen.

Eichkoff Kommunikation

Mareike Trippe

trippe@medizinmarketingservice.de
Boehringer Ingelheim. Patienten und Ärzten könnten in den kommenden Jahren neue Behandlungsalternativen für Atemwegserkrankungen zur Verfügung stehen. Boehringer Ingelheim stellte im November 2012 bei seiner 3. internationalen Pressekonferenz zu Forschung und Entwicklung (F\&E) seine umfassende Forschungs-Pipeline zur Behandlung chronisch obstruktiver Lungenerkrankungen (COPD), Asthma, idiopathischer Lungenfibrose sowie Lungenkrebs vor.

Boehringer Ingelheim

Arnd Prillip

presse@boehringer-ingelheim.de 